\title{
BMJ Open Coronavax: preparing community and government for COVID-19 vaccination: a research protocol for a mixed methods social research project
}

\author{
Katie Attwell (1) , ${ }^{1,2}$ Samantha Carlson, ${ }^{1,2}$ Jordan Tchilingirian (D) , ${ }^{1}$ Tauel Harper, ${ }^{3}$ \\ Lara McKenzie, ${ }^{1}$ Leah Roberts, ${ }^{1}$ Marco Rizzi (D) , ${ }^{4}$ Darren Westphal, ${ }^{5}$ Paul Effler, ${ }^{5,6}$ \\ Catherine Hughes, ${ }^{7}$ Valerie Swift, ${ }^{2}$ Christopher C Blyth ${ }^{2,8}$
}

To cite: Attwell K, Carlson S, Tchilingirian J, et al. Coronavax: preparing community and government for COVID-19 vaccination: a research protocol for a mixed methods social research project. BMJ Open 2021;11:e049356. doi:10.1136/ bmjopen-2021-049356

- Prepublication history and supplemental material for this paper is available online. To view these files, please visit the journal online. (http://dx.doi. org/10.1136/bmjopen-2021 049356).

Received 28 January 2021 Accepted 28 May 2021

Check for updates

(c) Author(s) (or their employer(s)) 2021. Re-use permitted under CC BY-NC. No commercial re-use. See rights and permissions. Published by BMJ.

For numbered affiliations see end of article.

Correspondence to

Dr Katie Attwell;

katie.attwell@uwa.edu.au

\section{ABSTRACT}

Introduction Ahead of the implementation of a COVID-19 vaccination programme, the interdisciplinary Coronavax research team developed a multicomponent mixed methods project to support successful roll-out of the COVID-19 vaccine in Western Australia. This project seeks to analyse community attitudes about COVID-19 vaccination, vaccine access and information needs. We also study how government incorporates research findings into the vaccination programme.

Methods and analysis The Coronavax protocol employs an analytical social media study, and a qualitative study using in-depth interviews with purposively selected community groups. Participant groups currently include healthcare workers, aged care workers, first responders, adults aged 65+ years, adults aged 30-64 years, young adults aged 18-29 years, education workers, parents/ guardians of infants and young children ( $<5$ years), parents/guardians of children aged $5-18$ years with comorbidities and parents/guardians who are hesitant about routine childhood vaccines. The project also includes two studies that track how Australian state and Commonwealth (federal) governments use the study findings. These are functional dialogues (translation and discussion exercises that are recorded and analysed) and evidence mapping of networks within government (which track how study findings are used).

Ethics and dissemination Ethics approval has been granted by the Child and Adolescent Health Service Human Research Ethics Committee (HREC) and the University of Western Australia HREC. Study findings will be disseminated by a series of journal articles, reports to funders and stakeholders, and invited and peer-reviewed presentations.

\section{INTRODUCTION}

The global scientific community has raced to develop effective and safe COVID-19 vaccines, yet vaccine development solves only half of the problem in dealing with the threat of the disease. Recent modelling suggests both high efficacy and coverage rates are essential, ${ }^{1}$ and a plan for roll-out is crucial. Experience
Strengths and limitations of this study

- This multicomponent mixed methods study employs substudies to understand community attitudes towards COVID-19 vaccination and explore how governments use research findings.

- It uses qualitative semistructured interviews; an appropriate methodology for exploring unexamined phenomena.

- The study engages with social media messaging, including TikTok, Instagram and other popular platforms.

- The novel functional dialogues methodology combines research translation with a social scientific exploration of expert and government collaboration during a crisis.

- Findings may not be generalisable, but this model is available for granular research in global settings.

gained from seasonal and pandemic influenza vaccination, ${ }^{2-4}$ as well as routine childhood vaccination, ${ }^{5}{ }^{6}$ demonstrates that building confidence and effective delivery is a science in itself. An effective COVID-19 vaccination programme will allow societies to resume economic, social and cultural function. This project assists in fulfilling that goal.

As of 12 May 2021, there were 99 vaccines in clinical development as recorded on the WHO COVID-19 candidate vaccine landscape database. ${ }^{7}$ Australia commenced its COVID-19 vaccination programme on 22 February 2021 with the Pfizer-BioNTech (Comirnaty) COVID-19 vaccine, and commenced administration of the AstraZeneca vaccine on 8 March 2021. Priority groups for vaccination have included quarantine and border workers, front-line healthcare workers (those in facilities such as intensive care units and emergency departments; laboratory staff handling potentially infectious material; ambulance 
and paramedic services; general practice respiratory clinics; and COVID-19 testing facilities), aged care and disability care staff (nursing and personal care staff; allied health professionals; kitchen, cleaning, laundry, garden and office staff), and aged care and disability care residents. ${ }^{8}$ The next priority group included all other healthcare workers, household contacts of quarantine and border workers, critical and high-risk workers (defence; police; fire; emergency services; meat processing), people aged $\geq 70$ years, Aboriginal and/or Torres Strait Islander people aged $\geq 50$ years and adults with an underlying medical condition or significant disability.

Australia's federal government developed the country's COVID-19 vaccination programme, ${ }^{9}$ shaped by a number of health and government bodies. Key among them is the country's national regulator, the Therapeutic Goods Administration, which is responsible for assessing the safety, quality and efficacy of vaccines, including any COVID-19 vaccines. The Australian Technical Advisory Group on Immunisation also provides technical input for a COVID-19 vaccination programme, tasked with identifying priority populations for vaccination. The federal government and the governments of the states and territories share responsibility for programme implementation, through national and local implementation plans.

Australia has experienced relatively little community transmission since the pandemic began, especially compared with culturally similar contexts like the USA, UK and Continental Europe. This is particularly the case in Western Australia (WA), which, until early February 2021, had no community transmission of the virus for nearly 10 months, ${ }^{10}$ and has had very little community transmission since. ${ }^{11}$ The country and its states and territories have implemented strict border controls, restricting visitors and enforcing 2-week quarantines. Australia, and WA within it, is therefore a unique place to study the public's views on and acceptance of COVID-19 vaccination. With relative safety, for instance, comes the possibility that people may delay or avoid vaccination. This unique Coronavax study is therefore necessarily sensitive to barriers and challenges to vaccination that may arise in Australia and globally.

Australians are generally highly supportive of vaccination, with $87 \%$ believing that vaccines are safe, effective and necessary ${ }^{12}$; less than $6 \%$ of Australians do not believe that vaccines are safe.$^{13}$ However, recent pandemic experience with influenza A/H1N109 saw poor vaccine uptake. One study found $26 \%$ of refusers were concerned about safety and $17 \%$ did not believe in the vaccine. ${ }^{14}$ Studies conducted between April and June 2020 found between $65 \%$ and $86 \%$ of Australians were likely to accept the COVID-19 vaccine. ${ }^{15-17}$ The WHO listed 'vaccine hesitancy' as one of the top threats to global health in $2019,{ }^{18}$ even before the pandemic. Hence, implementing an evidence-based plan for COVID-19 vaccine roll-out is crucial.

To facilitate an effective COVID-19 vaccination programme, scholarship needs to inform COVID-19 vaccine communications and to help develop effective mechanisms of governance for a vaccination programme. Governments must understand what communities require and identify the factors enabling widespread vaccine acceptance. A central aim of this project is therefore to facilitate communication between governments and members of the public. Communities are not homogenous, and they will need different messaging, encouragement and access based on their demographic features, values, locations and experiences of disease threat and lockdown. ${ }^{19}$ Building and maintaining trust is central, as mistakes can have lasting and devastating repercussions. Previous pandemic vaccine roll-outs demonstrate this; for example, in France, a challenging and rapidly implemented roll-out of the influenza A/ H1N1 vaccine united a heterogeneous front of vaccinehesitant populations. ${ }^{20}$ Engaging transparently and continuously with those who might undermine vaccination programmes through holding or disseminating negative views of vaccination is best practice in risk management, and increasingly important in the age of social media. ${ }^{21}$

The project facilitates communication and ensures shared understanding between communities and governments during COVID-19 vaccination roll-out. It uses interviews and dialogue to analyse and ensure uptake of the vaccine by specific subgroups, including those at greatest risk of disease or transmission of COVID-19. Both state and federal governments access findings, which has the potential to inform communication and governance recommendations for subgroups of Australians.

The project's objectives are:

1. To ascertain best practice and lessons for pandemic vaccination roll-outs from currently existing literature relating to pandemics, vaccine acceptance and cognate areas.

2. To ascertain the attitudes, beliefs, barriers, enablers and communication needs of key population subgroups regarding COVID-19 vaccination, and of the population more generally (through social media analysis).

3. To determine, through functional dialogues with government stakeholders, the feasibility of specific governance levers and the appropriate messaging for government communications about a COVID-19 vaccine.

4. To feed back findings and recommendations to government regularly and iteratively, while generating data on the experience of translating evidence into practice, and building a knowledge base for research and governance collaborations during crises.

\section{METHODS/ANALYSIS}

Coronavax has four main components: community interviews, social media analysis, functional dialogues and a study mapping governments' evidence uptake and use. 


\section{Social media study design, sample and analysis}

Social media is increasingly important as a source of information and disinformation for the broader public. The social media component of the study involves monitoring social media platforms used by local communities, in order to stay abreast of real-time concerns or controversies. This process will involve weekly searches for mentions of the COVID-19 vaccine in various social media channels and the collection of data based on those searches. It is not expected that these searches will comprehensively collect all mentions of the vaccine on social media, rather it is seen to be enough coverage to provide an overview of prominent and emerging concerns. This broad data collection will provide the basis for more targeted research about the origin and spread of concerns and controversies about the vaccine and the evolution of vaccine sentiment over time. The resulting data will be analysed using both discourse analysis and network analysis.

The type of data to be collected includes:

- Comments and posts made by social media users, pages and groups.

- Interactions with these messages, such as shares, retweets and reactions.

- Basic demographic data shared publicly on social media. Such data may include location, gender and age.

This aspect of the study collects data that may influence public sentiment. We will only use data from the public domain and include only publicly available posts and comments. The majority will be from search tools available to anyone-specifically Twitter, Instagram, TikTok, YouTube and Facebook's Search Console, Google Trends, Google Search and BoardReader (which searches publicly available discussion boards such as Reddit).

There may be some use of curated data in order to make the search for relevant data easier. For instance, Facebook have provided academic access to their 'CrowdTangle' application, allowing researchers on this project access to data on the spread of information via Facebook, Reddit, Instagram and Twitter. CrowdTangle allows us to monitor discussions in Facebook pages that are used by cultural and linguistic minority communities, as well as information provided by advocacy organisations through that platform. The data that Facebook releases via this application are only that which are publicly available. The access provided for this project collates data on posts with the highest number of public interactions. This does not include private Facebook groups or personal profiles.

Accounts established on Instagram, Twitter, TikTok and YouTube manually follow stories about the COVID-19 vaccine, accessed weekly in order to assess and code what sort of information about the vaccine each platform algorithmically selects as 'recommended' for these accounts. We do this by coding the first 10 stories or posts in each feed. The naming of these accounts makes it clear that they are research accounts, and they only view and follow COVID-19 vaccination stories and posts. The data collection schedule includes a weekly scrape of the top posts on each feed and platform. NVivo ${ }^{22}$ capture gathers data such as message content, source and engagement, which is then stored for analysis.

Social media data is analysed in two ways:

1. Discourse analysis: this involves looking at people's comments and overall concerns. Frameworks that used to code data and analyse discourse include, but are not limited to:

- Media type.

- Level of engagement.

- Stated concerns about the vaccine.

- Identifying where messages position themselves in terms of Leask et al's vaccination position hierarchy. ${ }^{23}$

- Broad message sentiment, rhetoric and composition.

- Typology of information spreader.

2. Network analysis: this involves examining the spread of messages and concerns. It includes understanding who holds credibility on social media, how ideas are amplified, what sorts of messages spread across what networks and what messages are spread between networks. Network analysis will also analyse how geographical and cultural issues of 'imagined communities' affect the spread of information and disinformation. This research has significant benefits in terms of monitoring and reporting the spread of information about COVID-19 vaccination programmes. It will also help improve understandings of how information spreads through social media networks. This knowledge will help guide public health communication during the roll-out of the coronavirus vaccine as well as informing future public communication programmes and initiatives. Network data will be analysed using UCINet ${ }^{24}$ and Statnet ${ }^{25}$ for R.

\section{Community interviews study design, sample and analysis}

The project involves semistructured in-depth interviews of approximately 1 hour with those living in WA. As determined by the research team in collaboration with the WA Department of Health, interviewees include those who need the vaccine first, but also those who possess the capacity to undermine a vaccination campaign through holding or disseminating negative views of vaccination. ${ }^{26} 27$ For each of the following participant groups there will be interviews with approximately 20 people (or fewer if achieving data saturation earlier):

1. People who work in healthcare (eg, doctors, nurses, paramedics and cleaners).

2. People who work in aged care (eg, doctors, nurses, paramedics, cleaners and carers).

3. Adults aged $65+$ years.

4. Adults aged $30-64$ years.

5. Young adults aged 18-29 years.

6. Education workers (ie, childcare and schoolteachers).

7. Parents/guardians of infants and young children $(<5$ years). 
8. Parents/guardians of children aged 5-18 years, with comorbidities.

9. Parents/guardians who are hesitant about routine childhood vaccines.

10. Aboriginal and Torres Strait Islander people in Perth.

11. Adults in regional or remote areas of WA.

12. Culturally and linguistically diverse people.

13. Adults with comorbidities.

14. Service delivery organisations (with lived expert representatives ${ }^{28}$ ) for especially vulnerable populations such as the homeless, drug dependent and victims of domestic violence. Methodologies for engagement with these groups will be developed based on best practice, including the use of translators and interpreters where necessary.

15. Pharmacists.

Participants discuss their experiences and views of the pandemic and lockdown period/s, as well as their views regarding COVID-19 vaccination in WA (see online supplemental appendix A for guiding questions, which may change over the course of data collection to capture contemporary issues or concerns). Participants answer additional questions depending on which categories they are in. A number of participants may belong to one or more of the categories, for instance, interviewee ' $\mathrm{X}$ ' might be an adult, working in aged care, who has a child under the age of 5 years. They would therefore receive generic adult questions with additional questions related to their occupation and being a parent to young child.

In order to ensure the sample includes the target groups and is as diverse as possible, participants are prescreened using Research Electronic Data Capture (REDCap) software. REDCap is a secure, web-based software platform designed to support data capture for research studies, providing (1) an intuitive interface for validated data capture; (2) audit trails for tracking data manipulation and export procedures; (3) automated export procedures for seamless data downloads to common statistical packages; and (4) procedures for data integration and interoperability with external sources. ${ }^{29} 30$ The prescreening survey first captures important demographic details that enable screening for particular types of participants, in addition to providing contact details and preferences for interviews days and times (see online supplemental appendix B). Participants then have the option of continuing to a follow-up survey in REDCap, or of answering these follow-up questions during their interview. Questions in the second survey capture important information about participants that are not necessary for the purposes of screening (see online supplemental appendix $\mathrm{C}$ ).

Brochures, posters, word of mouth and media releases (online supplemental appendix D) distribute the prescreening survey in the Perth metropolitan area (figure 1). The design of posters and brochures was in collaboration with the Telethon Kids Institute (TKI) communications and graphic designer team. Recruitment also draws on investigators' existing networks. If recruitment through community-based methods is insufficient, there will be further recruitment through government and non-government workplaces and institutions (subsequent to ethics approval) (figure 2).

A number of groups, such as parents/guardians who are hesitant and/or resistant to childhood vaccines, may be difficult to access. However, KA has previously worked with such parents. ${ }^{31}$ They will be recruited through the channels described above at 2.2, with an emphasis on the use of personal networks, maintaining confidentiality and providing distance from personal relationships (other interviewers will conduct these interviews).

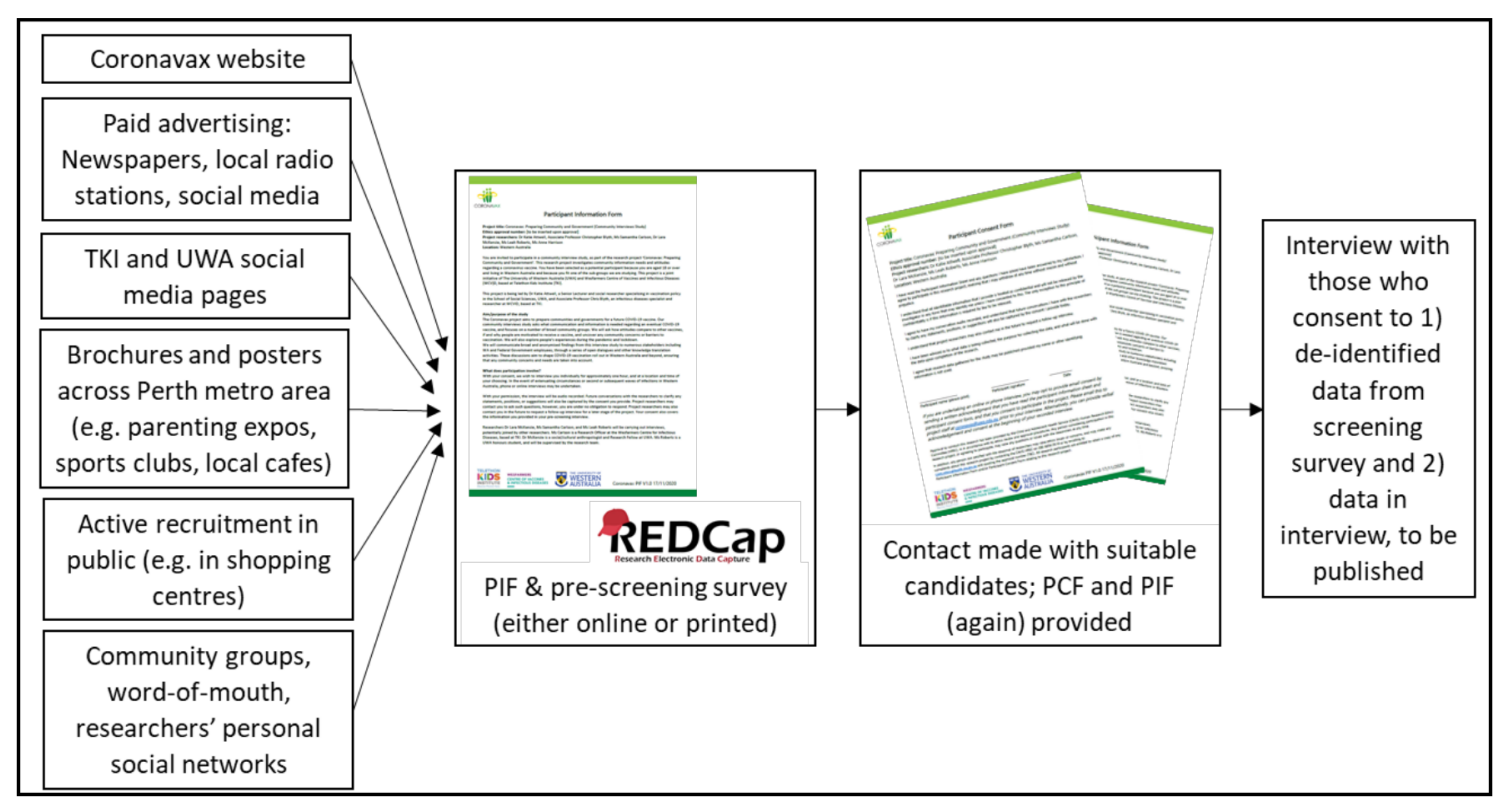

Figure 1 Steps involved in community recruitment strategy. PCF, Participant Consent Form; PIF, Participant Information Form; TKI, Telethon Kids Institute; UWA, University of Western Australia. 




Figure 2 Steps involved in institutional recruitment strategy. PCF, Participant Consent Form; PIF, Participant Information Form.

Information and consent materials for the project explain the research's basis in improving vaccine uptake. This provides transparency for participants, who can take the opportunity to share their critiques and concerns through the research as they have through KA's previous work.

Research with Aboriginal and/or Torres Strait Islander people should be led by Aboriginal and/or Torres Strait Islander people and communities. The research team must include Aboriginal and/or Torres Strait Islander members, and the priorities should be guided by the community with whom the researchers intend on recruiting within. ${ }^{32}$ Thus, to assist with recruitment and interpretation of the views of Aboriginal people in Perth (as per the WA Aboriginal Health and Well-being Framework, ${ }^{33}$ 'Aboriginal' is the preferred term over 'Aboriginal and/or Torres Strait Islander' in WA), an Aboriginal cultural guidance advisor and researcher has been engaged (VS). With the recent awarding of additional funding (see the Funding statement section), we are employing an Aboriginal researcher to undertake extensive community consultation and to later conduct interviews in a culturally sensitive fashion with this priority group for COVID-19 vaccination in Australia. ${ }^{34}$ These interviews will be conducted with Aboriginal people in Perth; this community predominantly uses English as their main language spoken at home. ${ }^{35}$ Research with Aboriginal people in regional areas remains in the project plan.

Interviews may be conducted by video chat, phone or in person. For the bulk of participant groups, face-toface interviews are the preferred method, dependent on COVID-safe policies and recommendations at the time of interviews. For people less eager to participate in face-toface interviews or who face technological barriers, alternative methods are employed.

Thematic analysis of the data uses NVivo V.12 software. ${ }^{22}$ This analytical process will be collaborative, with inductive themes decided jointly by the research team based on research questions, and then revisited throughout the analysis deductively with the emergence of new themes. Analysis will follow the steps outlined in the Braun and
Clarke method ${ }^{36}$ If post-interview clarifications or additional information is required, investigators may follow-up with participants (eg, by email). Data collected in these follow-up conversations may be included in the project findings.

\section{Functional dialogues study design, sample and analysis}

Coronavax is centrally concerned with vaccine governance. Regular functional dialogues (a novel two-way qualitative data gathering and information exchange meetings) take place with relevant government departments and committees within WA and Australia. These : (1) study how policymakers understand and implement the tools available to best drive vaccine uptake; (2) ensure rapid dissemination of emerging insights from ongoing fieldwork to stakeholders in WA and federal governments; and (3) encourage stakeholders and the research team to shape fieldwork through collaboration.

Investigators work with government partners to identify key individuals who: (1) have used or will use the emerging findings, and (2) can speak on behalf of their team/department, including in terms of providing feedback to inform the ongoing conduct of each Coronavax component. Researchers from the Coronavax research team are selectively included when discussing relevant findings or project components.

A facilitator from the research team leads each functional dialogue (JT). Conversations follow a semistructured discussion framework, giving time for research dissemination and in-depth conversations between researchers and stakeholders while exploring stakeholders' attitudes, beliefs, experiences, roles and observations in detail. Meetings with local participants will be face to face, or via online videoconferencing if it is not COVID safe to meet face to face. Functional dialogues with federal participants are via videoconferencing. This is necessary in light of current internal (state and territory) border closures and travel restrictions within Australia. 
Mapping evidence uptake and use study design, sample and analysis

Coronavax's interest in vaccine governance will also focus on knowledge sharing by government stakeholders and policymakers. To do so, investigators will conduct a quantitative social network analysis of WA state government employees intra/inter stakeholder information-sharing networks recruited through the functional dialogues (above). Social network analysis enables the research team to both visualise the structure of stakeholders and analyse the properties of these relationships. This enables investigators to understand how evidence travels through government. This has two benefits: (1) identifying blocks to communication, and (2) helping stakeholders to redesign their communication networks to achieve more effective working practices.

Data collection is through a short nine-item structured questionnaire, administered by hand and online using Qualtrics survey software ${ }^{37}$ (online supplemental appendix E). Themes in the questionnaire include knowledge, understanding and use of Coronavax findings; internal colleagues with whom the participant discusses COVID-19 vaccine roll-out; and external colleagues with whom the participant discusses COVID-19 vaccine roll-out. Data gathered through the questionnaire will be analysed using the specialist social network analysis software UCINet. Investigators will explore the overall structure of the communication network as well as the existence of any structurally important actors in the network (such as those who are central or who bridge groups and clusters). Part of the discussion will focus on evidence-sharing networks. Using these suggested connections, the research team will be able to 'snowball' and identify relevant departments/teams as potential respondents to the survey. ${ }^{38}$

\section{Patient and public involvement}

Consumers (led by $\mathrm{CH}$ ) have informed the project's design, which has been undertaken with three main goals in mind:

1. Ensure the appropriateness and sensitivity of research questions and recruitment.

2. Ensure that the research priorities and design reflect the needs of the community.

3. Explicitly bring the consumer voice back to government as part of the functional dialogues.

Involving advocates and trained consumer representatives and related organisations, such as the Immunisation Foundation of Australia and 'Light for Riley' online vaccine advocacy campaign, is central to the project's community engagement strategy. Consumers (led by $\mathrm{CH})$ advise on the community interviews and contribute to all sections of the protocol. This approach is supported by a network of consumer involvement experts from the TKI and the Consumer and Community Health Research Network, including the Wesfarmers Centre of Vaccines and Infectious Diseases Community Reference Group (chaired by $\mathrm{CH}$ ). This community reference group consulted on Coronavax in September 2020.

\section{ETHICS AND DISSEMINATION}

This project has ethical approval from the Child and Adolescent Health Services Human Research Ethics Committee (HREC) under permit number RGS0000004457. The University of Western Australia (UWA) HREC reciprocally recognised this approval under permit number 2020/ ET000339, and separately granted ethical approval for the federal functional dialogues under permit number 2020/ET000340. Our protocol has undergone three amendments with more pending to include new community study cohorts for which we have attained additional funding. These have been explicitly detailed above.

Participants and (where relevant) their workplaces or institutions are given pseudonyms, ensuring that their anonymity is respected in any communications and publications arising from this research. Publications and presentations will omit specific information that may identify participants. Participant data are manually reidentifiable and will only be accessed and analysed by the researchers.

Identifying details collected in the prescreening survey of the community interviews are stored on REDCap. All audio transcripts created during the community interviews and functional dialogues are stored temporarily with Otter and permanently deleted following transcription. ${ }^{39}$ Audio files collected in the community interviews and functional dialogues, transcripts, evidence-mapping survey data and any other non-identifying information are stored under associated pseudonyms on UWA's Institutional Research Data Store (IRDS). IRDS supports the requirements of researchers regarding confidentiality, integrity, availability, security and ownership of data. ${ }^{40}$ Investigators will keep the project data on UWA REDCap and UWA IRDS (for 7 years after date of final publication, or 7 years after conclusion of the project, whichever is later). All hard copy documents obtained during the research (eg, paper questionnaires, consent forms) will be stored in a secure filing cabinet in the lead author's office. Investigators will securely destroy them at the time of electronic data disposal.

Investigators anticipate publishing several articles from each Coronavax component in peer-reviewed journals that publish articles on vaccines; infectious diseases; medicine; public health; social sciences; relationships; family; employment; anthropology; gender; and health sociology. Project investigators will also share results more widely, including with and through the media, as well as by presenting at conferences and seminars.

\section{Author affiliations}

${ }^{1}$ School of Social Sciences, The University of Western Australia, Perth, Western Australia, Australia

${ }^{2}$ Wesfarmers Centre of Vaccines and Infectious Diseases, Telethon Kids Institute, Nedlands, Western Australia, Australia 
${ }^{3}$ Faculty of Arts, Business, Law and Education, The University of Western Australia, Perth, Western Australia, Australia

${ }^{4}$ School of Law, The University of Western Australia, Perth, Western Australia, Australia

${ }^{5}$ Communicable Disease Control Directorate, Department of Health Government of Western Australia, Perth, Western Australia, Australia

${ }^{6}$ Faculty of Health and Medical Sciences, The University of Western Australia, Perth, Western Australia, Australia

${ }^{7}$ Immunisation Foundation of Australia, Perth, Western Australia, Australia

${ }^{8}$ Wesfarmers Centre of Vaccines and Infectious Diseases, The University of Western Australia, Perth, Western Australia, Australia

\section{Twitter Jordan Tchilingirian @JordanTchil}

Acknowledgements The team thanks the Wesfarmers Centre of Vaccines and Infectious Diseases (WCVID) Community Reference Group for consulting on this protocol, and Sian Tomkinson and Shevaun Drislane for their assistance with preparing the manuscript for submission. We acknowledge Stacey Campbell and the wider communications team from Telethon Kids Institute for their dedication spent in working with us to design the recruitment resources for the community interviews.

Contributors KA conceptualised the project, convened and managed the research team and worked in collaboration with each component lead to design the project. She also led the research funding applications and wrote the core sections of the protocol as well as editing and drafting this protocol manuscript. SC was responsible for writing the core sections of the protocol, collated and managed the contributions of coauthors to the protocol, contributed to the writing of funding applications, managed the HREC application process, as well as drafting and editing this protocol manuscript. JT wrote the core sections of the protocol, designed the functional dialogues and evidence uptake and use mapping, contributed to the development of the social media study and managed the ethics applications to UWA HREC application for functional dialogues with federal policymakers. TH wrote the core sections of the social media study and had input on the functional dialogues section. He is the research team leader for the social media study. LM led the design of the community interviews with KA and SC, cowrote the relevant sections of the protocol and contributed to the editing of this manuscript. LR assisted with the design of the community interviews and contributed to the editing of this manuscript, including preparing the appendices. MR contributed to the conceptualisation of the project with regard to the role of the government in vaccine roll-out, the development of the functional dialogues with JT and he assisted with the ethics application to UWA HREC. DW provides institutional liaison with the Department of Health, Western Australia, and has contributed to the project design. He contributed to the editing of this manuscript. PE represents with the Department of Health, Western Australia, and has contributed to the project design from its inception. CH contributed to the project's consumer involvement plan, and provided consumer insight relevant to the protocol, participant information sheets, participant recruitment materials, community interview questions and social media project. VS, an Aboriginal woman and Aboriginal cultural governance advisor to the Wesfarmers Centre of Vaccines and Infectious Diseases, Telethon Kids Institute, provided leadership and guidance to ensure this project addresses the needs of First Nations families. She continues to shape the team's culturally secure recruitment and engagement for its ongoing research programme. CCB has contributed significant medical, technical and research expertise to all aspects of this project. He has led funding applications and provided resources, engaged in high-level conceptualisation of the project in its entirety and led key aspects of its stakeholder engagement. He contributed to the writing of the protocol as well as the drafting and editing of this protocol manuscript. All authors contributed to the editing of this manuscript.

Funding Funding for this project was provided by a Wesfarmers Centre of Vaccines and Infectious Diseases 2020 Catalyst Research Grant (WCVID Catalyst Grant 2020. Coronavax: Preparing Community and Government) and the Government of Western Australia's Department of Health (DOH2020-6075) A subsequent Future Health Research Innovation grant from the Government of Western Australia (FHRIFGCOVID19) funds additional community sub-group interviews. KA is funded by the Australian Research Council of the Australian Government under DEDE190100158. She has previously received a speaker's fee from Merck.

Disclaimer The funding providers, organisations involved in the study and individual members of the research team have no financial interest in the outcome of the research. Government funding partners will contribute to the research question design and contribute to the functional dialogues, although not to their research design. All funders will also participate in the dissemination of research findings.

Competing interests None declared.

Patient consent for publication Not required.

Provenance and peer review Not commissioned; externally peer reviewed.

Supplemental material This content has been supplied by the author(s). It has not been vetted by BMJ Publishing Group Limited (BMJ) and may not have been peer-reviewed. Any opinions or recommendations discussed are solely those of the author(s) and are not endorsed by BMJ. BMJ disclaims all liability and responsibility arising from any reliance placed on the content. Where the content includes any translated material, BMJ does not warrant the accuracy and reliability of the translations (including but not limited to local regulations, clinical guidelines, terminology, drug names and drug dosages), and is not responsible for any error and/or omissions arising from translation and adaptation or otherwise.

Open access This is an open access article distributed in accordance with the Creative Commons Attribution Non Commercial (CC BY-NC 4.0) license, which permits others to distribute, remix, adapt, build upon this work non-commercially, and license their derivative works on different terms, provided the original work is properly cited, appropriate credit is given, any changes made indicated, and the use is non-commercial. See: http://creativecommons.org/licenses/by-nc/4.0/.

\section{ORCID iDs}

Katie Attwell http://orcid.org/0000-0002-0366-2160

Jordan Tchilingirian http://orcid.org/0000-0002-9509-3638

Marco Rizzi http://orcid.org/0000-0003-0445-9655

\section{REFERENCES}

1 Bartsch SM, O'Shea KJ, Ferguson MC, et al. Vaccine efficacy needed for a COVID-19 coronavirus vaccine to prevent or stop an epidemic as the sole intervention. Am J Prev Med 2020;59:493-503.

2 Carlson SJ, Quinn HE, Blyth CC, et al. Barriers to influenza vaccination of children hospitalised for acute respiratory illness: a cross-sectional survey. J Paediatr Child Health 2021;57:409-18.

3 King CL, Chow MYK, Wiley KE, et al. Much ado about flu: a mixed methods study of parental perceptions, trust and information seeking in a pandemic. Influenza Other Respir Viruses 2018:12:514-21.

4 Blyth CC, Richmond PC, Jacoby P, et al. The impact of pandemic A(H1N1)pdm09 influenza and vaccine-associated adverse events on parental attitudes and influenza vaccine uptake in young children. Vaccine 2014;32:4075-81.

5 Attwell K, Dube E, Gagneur A, et al. Vaccine acceptance: Science, policy, and practice in a 'post-fact' world. Vaccine 2019;37:677-82.

6 Attwell K, Leask J, Meyer SB, et al. Vaccine rejecting parents' engagement with expert systems that inform vaccination programs. $J$ Bioeth Inq 2017;14:65-76.

7 World Health Organization. Draft landscape and tracker of COVID-19 candidate vaccines, 2021. Available: https://www.who. $\mathrm{int} /$ publications $/ \mathrm{m} /$ item/draft-landscape-of-covid-19-candidatevaccines [Accessed May 2021].

8 Australian Government Department of Health. When will I get a COVID-19 vaccine? 2021. Available: https://www.health.gov.au/ initiatives-and-programs/covid-19-vaccines/getting-vaccinated-forcovid-19/when-will-i-get-a-covid-19-vaccine [Accessed May 2021].

9 Australian Government. Australian COVID-19 vaccination policy. Canberra: Government of Australia, 2020. https://www.health.gov. au/sites/default/files/documents/2020/12/australian-covid-19vaccination-policy.pdf

10 Premier of Western Australia Mark McGowan. - Important Update Regarding Covid-19 In Western Australia, and Perth, Peel and South-west Five-day Lockdown, 2021. Available: https://www. facebook.com/permalink.php?story_fbid $=5260057527345412 \& \mathrm{id}=$ 426913580659855 [Accessed May 2021].

11 Premier of Western Australia Mark McGowan. Nine months without community transmission of COVID, 2021. Available: https://m facebook.com/MarkMcGowanMP/photos/a.432761316741748/ 5168572266493939/?type=3\&source=48 [Accessed May 2021].

12 Smith DT, Attwell K, Evers U. Majority acceptance of vaccination and mandates across the political spectrum in Australia. Politics 2020;40:189-206.

13 Larson HJ, de Figueiredo A, Xiahong Z, et al. The state of vaccine confidence 2016: global insights through a 67-country survey. EBioMedicine 2016;12:295-301. 
14 Eastwood K, Durrheim DN, Jones A, et al. Acceptance of pandemic (H1N1) 2009 influenza vaccination by the Australian public. Med J Aust 2010;192:33-6.

15 Dodd $\mathrm{RH}$, Cvejic E, Bonner $\mathrm{C}$. Willingness to vaccinate against COVID-19 in Australia. Lancet Infect Dis 2020;21.

16 Rhodes A, Hoq M, Measey M-A, et al. Intention to vaccinate against COVID-19 in Australia. Lancet Infect Dis 2021;21:e110.

17 Attwell K, Lake J, Sneddon J, et al. Converting the maybes: crucial for a successful COVID-19 vaccination strategy. PLoS One 2021;16:e0245907.

18 World Health Organization. Ten threats to global health in 2019. Geneva: World Health Organization, 2019. https://www.who.int/ news-room/spotlight/ten-threats-to-global-health-in-2019

19 Nowak GJ, Gellin BG, MacDonald NE, et al. Addressing vaccine hesitancy: the potential value of commercial and social marketing principles and practices. Vaccine 2015;33:4204-11.

20 Ward JK. Rethinking the antivaccine movement concept: A case study of public criticism of the swine flu vaccine's safety in France. Soc Sci Med 2016;159:48-57.

21 Lundgren RE, McMakin AH. Risk communication. Newark: John Wiley \& Sons, Incorporated, 2018.

22 Nvivo12 Qualitative Data Analysis Software [program]: SAGE Ocean 1999.

23 Leask J, Kinnersley P, Jackson C, et al. Communicating with parents about vaccination: a framework for health professionals. BMC Pediatr 2012;12:154.

24 Ucinet for Windows. Software for Social Network Analysis (6.389) [program]. Harvard, MA: Analytic Technologies, 2002.

25 statnet. Software tools for the Statistical Modeling of Network Data [program]. Statnet, 2003.

26 Ward JK. Rethinking the antivaccine movement concept: a case study of public criticism of the swine flu vaccine's safety in France. Soc Sci Med 2016;159:48-57.

27 Raude J, Caille-Brillet A-L, Setbon M. The 2009 pandemic H1N1 influenza vaccination in France: who accepted to receive the vaccine and why? PLoS Curr 2010;2:RRN1188-RRN88.

28 Videmšek P. Expert by experience research as grounding for social work education. Social Work Education 2017;36:172-87.
29 Harris PA, Taylor R, Minor BL, et al. The REDCap Consortium: building an international community of software platform partners. $J$ Biomed Inform 2019;95:103208-08.

30 Harris PA, Taylor R, Thielke R, et al. Research electronic data capture (REDCap)--a metadata-driven methodology and workflow process for providing translational research informatics support. J Biomed Inform 2009;42:377-81.

31 Attwell K. The politics of picking: selective vaccinators and population-level policy. SSM Popul Health 2019;7:100342.

32 National Health and Medical Research Council. Ethical conduct in research with Aboriginal and Torres Strait Islander peoples and communities, 2018. Available: www.nhmrc.gov.au/guidelinespublications/ind2 [Accessed May 2021].

33 Government of Western Australia Department of Health. Wa Aboriginal health and wellbeing framework 2015-2030, 2015 Available: https://ww2.health.wa.gov.au/Improving-WA-Health/ About-Aboriginal-Health/WA-Aboriginal-Health-and-WellbeingFramework-2015-2030 [Accessed May 2021].

34 Australian Technical Advisory Group on Immunisation (ATAGI). COVID-19 vaccination prioritisation. Canberra: Australian Government Department of Health, 2020. https://www.health.gov.au/ covid-19-vaccination-prioritisation

35 Australian Bureau of Statistics. Census of population and housing: characteristics of Aboriginal and Torres Strait Islander Australians, 2018. Available: https://www.abs.gov.au/statistics/people/aboriginaland-torres-strait-islander-peoples/census-population-and-housingcharacteristics-aboriginal-and-torres-strait-islander-australians/ latest-release\#language [Accessed May 2021].

36 Braun V, Clarke V. Using thematic analysis in psychology. Qual Res Psychol 2006;3:77-101.

37 Qualtrics survey software [program]. January 2021 version. Utah, USA: Qualtrics 2005

38 Knoke D, Yang S. Social network analysis: SAGE publications, 2019.

39 Otter.ai. Otter [online]. California, 2020. Available: https://otter.ai/ [Accessed Dec 2020].

40 The University of Western Australia. Institutional research data store. Perth, Australia, 2020. https://www.it.uwa.edu.au/service-catalogue/ data-storage/irds 